Sociologie et sociétés

\title{
Itinéraire d'une pratique de recherche sociale appliquée
}

\section{Yvan Corbeil : Une traversée exemplaire}

\section{Yvan Corbeil: An Exemplary Crossing}

\section{Roger TESSIER}

Volume 20, numéro 1, printemps 1988

La sociologie hors université

URI : https://id.erudit.org/iderudit/001789ar

DOI : https://doi.org/10.7202/001789ar

Aller au sommaire du numéro

Éditeur(s)

Les Presses de l'Université de Montréal

ISSN

0038-030X (imprimé)

1492-1375 (numérique)

Découvrir la revue

\section{Citer cet article}

TESSIER, R. (1988). Itinéraire d'une pratique de recherche sociale appliquée : yvan Corbeil : Une traversée exemplaire. Sociologie et sociétés, 20(1), 141-151. https://doi.org/10.7202/001789ar d'utilisation que vous pouvez consulter en ligne. 


\title{
Itinéraire d'une pratique de recherche sociale appliquée
}

\author{
Yvan Corbeil: Une traversée exemplaire
}

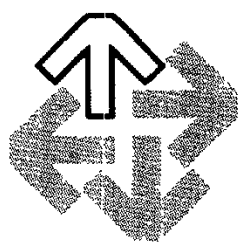

ROGER TESSIER

De la sociologie québécoise contemporaine, l'image qui s'impose est celle d'une discipline universitaire, dont l'histoire est écrite par les professeurs et les chercheurs universitaires. L'on connait peu et mal ce qui s'est fait et se fait en dehors des murs de l'université. Parce qu'ils utilisent rarement les canaux conventionnels de communication (articles dans des revues, communications à des congrès ou sociétés savantes), nous n'avons guère de «traces» des activités de ceux et celles qui, par engagement social ou au hasard des opportunités d'emploi, une fois diplômés (en sociologie, en service social ou en anthropologie), se sont orientés vers la «recherche sociale» ou ce qu'il est convenu d'appeler les «sciences sociales appliquées». Hier, le lieu privilégié d'exercice de ces activités - souvent plus près de l'intervention que de la recherche - était le gouvernement et les divers organismes paragouvernementaux (du BAEQ aux CLSC). Aujourd'hui, à la faveur du néo-libéralisme et d'un contexte où l'accès au marché du travail - surtout aux postes offrant une sécurité d'emploi - est difficile, les diplômés hésitent moins à frapper aux portes des entreprises privées. Ils veulent fuir la «grisaille» de la bureaucratie gouvernementale ou la soi-disant morosité des milieux universitaires. Certains se lancent même «en affaires» et mettent sur pied leur propre bureau de consultation; d'autres acceptent de travailler à la pige.

Ces «nouveaux entrepreneurs» en sciences sociales ne sont pas les premiers à affronter le marché; ils ont eu des précurseurs. La «recherche sociale» a ses pionniers. En raison de sa relative jeunesse, le Québec offre, pour celui qui veut écrire l'histoire d'une discipline ou d'une spécialité, le rare avantage de pouvoir interroger les pionniers!

Dans un numéro consacré à la "sociologie appliquée», la revue Sociologie et sociétés se devait de parler de ou de faire parler ceux et celles qui, les premiers, se sont engagés dans cette voie de recherche et d'action. Le choix d'Yvan Corbeil s'est rapidement imposé. Ce fils d'entrepreneur a choisi, au milieu des années 1950, de se marginaliser en se dirigeant vers des études en sciences sociales. Il est devenu doublement marginal, en marge à la fois du monde des affaires et du milieu universitaire. Position inconfortable: face aux représentants de l'industrie et du commerce, il est un intellectuel et un utopiste et face aux professeurs d'université, un homme d'affaires attiré par l'appât du gain. Mais aux yeux de tous, il a réussi. Son itinéraire permet de mieux comprendre les conditions (et les limites: obstacles et difficultés) d'exercice d'une «pratique» en sciences sociales. 
Nous avons demandé à Roger Tessier, psychosociologue et professeur au Département de communications à l'UQAM, de réaliser l'entrevue avec Yvan Corbeil. Sa formation, son itinéraire professionnel et ses liens d'amitié l'autorisent à accomplir la tâche délicate d'interroger ce spécialiste des sondages, à un moment difficile de sa vie. Nous avons respecté la volonté de l'interviewé: nous l'avons fait parler de sa vie professionnelle, il nous a aussi parlé de luimême, face à la mort. Sociologie et sociétés publie cet entretien afin de mieux faire connaître les idées et les actions de ce pionnier des sciences sociales appliquées que les nombreuses et diverses occupations ont éloigné de l'écriture. Nous saisissons aussi le prétexte pour lui rendre hommage.

M. F.

Cet article veut raconter la sociologie appliquée à travers l'histoire de vie d'un de ses plus illustres représentants. Sous plusieurs aspects, la biographie d'Yvan Corbeil correspond à celle d'un pionnier de la recherche sociale au Québec et au Canada: participation dès sa jeune vingtaine aux toutes premières recherches sociales faites au pays, au sein du Groupe de recherche sociale; mise sur pied de la première entreprise privée canadienne entièrement vouée à la recherche sociale sous toutes ses formes (le Centre de recherche sur l'opinion publique: C.R.O.P.); premier québécois ou canadien à occuper un rôle proéminent au plan international sur la scène de la recherche sur l'opinion publique [présidence de W.A.P.O.R. (World Association for Public Opinion Research)]. Mais c'est encore plus par certains apports contemporains que la carrière d'Yvan Corbeil se doit d'être donnée en exemple d'une manière «autre» - c'est-à-dire différente des usages académiques - de pratiquer la recherche sociale.

Par le souci de rigueur et la valorisation de la précision méthodologique au niveau de l'enquête empirique, la pratique d'Yvan Corbeil est tout ce que l'on peut imaginer de scientifique. Par contre, l'urgence d'agir, la liberté de manœuvre, le choix extrêmement varié des valeurs à servir, des actions à mener, représentent une certaine manière de faire de la recherche comportant des différences avec les us et coutumes proprement universitaires. Dès notre première entrevue, Yvan fait état de deux anecdotes qui, à elles seules, expriment très bien la marginalité où il s'est trouvé toute sa vie, ou presque, par rapport à l'univers académique:

Ma relation avec les canons officiels de l'Institution est en 1988 ce qu'elle était dès 1960. Avec quelques collègues du Service de recherche de la Société Radio-Canada, nous avions, à l'occasion du premier Festival international du film de Montréal, sollicité une petite subvention de cinq cents dollars au ministère des Affaires culturelles en vue d'étudier l'auditoire de cette première grande manifestation du cinéma à Montréal. Nous avons essuyé un refus et avons dû procéder à cette enquête avec les moyens du bord. Vingt-huit ans plus tard - autour d'une question qui me tient particulièrement à cœur, puisque je suis atteint d'une maladie très grave, la sclérose amyotrophique latérale, cloué à une chaise roulante - j'ai fait application au Conseil québécois de la recherche sociale demandant des fonds minimes pour mener une enquête sur l'adaptation des personnes handicapées à leur environnement physique et social. Encore là, je n'ai pas obtenu cette subvention. Ma demande ne comportait pas un nombre suffisant de références à la littérature établie et, pire encore, je n'avais pas étayé le budget sur l'ensemble des rubriques prévues par le formulaire. Je n'ai pu évidemment m'empêcher de sourire avec ironie à un tel retour des choses. Décidément, je n'aurai pas eu le profil académique!

Profil académique ou pas, la longue histoire professionnelle d'Yvan Corbeil demeure pour tous ceux qui ont à cœur les sciences sociales appliquées et leur pratique dans le monde contemporain, une traversée tout à fait exemplaire. Une traversée de l'époque, où, à travers vents et marées, Yvan sera demeuré fidèle à lui-même, c'est-à-dire à certaines valeurs fondamentales que les diverses mutations de son identité n'auront fait qu'épurer et rendre plus actives. Traversée également des institutions, de celles particulièrement opaques et contraignantes de la fin des années 50 au Québec; mais de celles aussi, plus flexibles, malgré tout envahissantes, d'une certaine modernité. Plus fondamentalement encore, cette traversée aura été celle des illusions. Traversée des illusions collectives sans doute très largement permise par la lucidité qu'une manière empirique d'envisager le social apporte à celui qui s'y donne pleinement. 
Traversée également d'illusions plus intimes qui ont trait à la vie, à la mort, au travail, à l'amour, et que paradoxalement, la vulnérabilité de la maladie alimente d'une manière fort importante.

Les illusions, qu'avez-vous contre? Ce sont elles qui font bouger la vie. La puissance spirituelle transforme les illusions en force de l'âme. (Jean Sulivan, in La traversée des illusions, Gallimard, 1977.)

\section{DE LA J.E.C. À RADIO-CANADA: L'APPRENTISSAGE DU MÉTIER DE CHERCHEUR.}

En 1950, Yvan Corbeil est étudiant au Collège Bourget de Rigaud. Son père a favorisé ce choix pour qu'il ne ressemble pas à la jeunesse d'Outremont. Au collège, c'est surtout la J.E.C. qui paraît à Yvan une expérience marquante. Bien sûr, grâce à l'appui du Père Telmosse et, encore là, à la complicité bienveillante de son père, Yvan, dès l'âge de quinze ans, en tant que cadet de l'armée canadienne, fait le tour du Canada, traverse les Prairies et séjourne dans les Rocheuses. Mais c'est surtout la J.E.C. qui retient son attention et il s'y consacre avec toute son énergie d'adolescent en donnant à son engagement une tournure activiste, au début:

À mon époque, quelqu'un qui vendait dans un collège de cinq cents étudiants, vingt abonnements à «Vie étudiante» était considéré comme un vendeur respectable. Or, j'ai mis au point une stratégie de vente qui fait que j'ai abonné toute la division des grands à «Vie étudiante», ce qui constituait un record sans précédent. Par contre, sous l'influence du Père Robert, l'aumônier de la section locale du Collège Bourget, je m'en vais rapidement vers une conception plus réfléchie de l'engagement militant: c'est surtout cette influence du Père Robert dont je garde un souvenir impérissable.

En 1954, Yvan se joint à l'équipe nationale de la Jeunesse étudiante catholique à titre de responsable du secteur des collèges classiques. Deux grands traits marqueront cette époque de son développement. Il adopte un point de vue personnaliste dont l'expression théorique se trouve surtout dans les travaux du grand philosophe français Emmanuel Mounier mais qui infiltre toute la vie d'Yvan et de ses collègues de l'époque:

À la J.E.C., j'ai appris à penser personnellement et à défendre un point de vue où l'expérience personnelle prime sur les croyances collectives et les règles de l'institution. J'y rencontrai, en plus, des jeunes de mon âge, dont certains demeureront toute ma vie de grands amis. Un homme qui m'aura marqué profondément: le Père Jean-Marc Chicoine, a été en quelque sorte, mon mentor et mon éveilleur intellectuel. C'est là aussi que pour la première fois, je m'intéresse au rapport entre la recherche sociale et l'action. L'Action catholique, vers la fin des années 50 , et particulièrement la J.E.C., ont donné certains contrats de recherche au Groupe de recherche sociale et à certains de ses invités provenant de l'École de sociologie de Chicago. Paule Verdet et Sally Casseday mèneront une enquête sur les catégories mentales des jeunes étudiants canadiens-français.»

Les élites intellectuelles de l'époque, au Québec et ailleurs, s'adonnent facilement à la spéculation ésotérique. Les projets collectifs sont imbus d'une idéologie, souvent noble et généreuse, mais dont les références concrètes sont plutôt fragiles.

Nous irons plus loin que le "voir» de la méthode de l'Action catholique: «Voir, Juger, Agir». Nous ne nous satisferons pas des impressions et des perceptions subjectives. Nous découvrons avec enthousiasme la science, son appareil méthodologique et son souci de rigueur. À partir de là, les problématiques abordées par la J.E.C. dans ses programmes devront s'étayer sur des faits: ces faits, ce sont des enquêtes systématiques qui les révéleront.»

Apparaît ainsi une première génération d'intellectuels activistes, soucieux de changements, de réforme, qu'on retrouvera un peu plus tard à Radio-Canada, à l'O.N.F., dans le syndicalisme, le mouvement coopératif ou l'éducation des adultes, constituant un important réservoir où s'alimentera un nouveau groupe social, la technocratie orientée vers la modernisation du Québec. On ne saurait sous-estimer son poids dans la mise en orbite de la révolution tranquille du début des années 60 . 
Pendant qu'il est membre de l'Équipe nationale de la J.E.C., Yvan prend contact avec le Mouvement des relations humaines américain, participe à des sessions à Bethel au National Training for Group Development, prend contact avec des intellectuels américains qui seront d'importantes références durant cette première étape: Robert Blake, Jane Mouton, Robert Bales, Fred Borgatta, Harold Leavitt, Gordon Lippitt. C'est là qu'il rencontre aussi Max et Robert Pagès, deux français en stage à Bethel comme lui avec lesquels il gardera contact.

Au cours de l'année 1956, il paraît évident aux membres de l'Équipe nationale, qu'ils ne feront plus long feu à la Centrale compte tenu de profondes dissensions qui les opposent aux institutions cléricales. La volonté de donner un contenu empirique à l'action, par-delà les slogans pieux, le consentement à la matérialité des choses, même dans le domaine social, parviennent à effrayer la hiérarchie et ses représentants auprès des équipes de direction du mouvement. À l'égalitarisme et à l'ouverture des aumôniers des années cinquante - les Pères Lafond, Brodeur, Chicoine, limogés sans cérémonie - succèdera un régime autoritaire et suspicieux ayant recours à la surveillance et à la délation. Un tel climat de méfiance rend tout travail impossible.

Un à un, les dirigeants démissionnent et Yvan quitte Montréal pour un long séjour à Toronto.

Mon père m'a prêté cinquante dollars et je suis descendu chez des amis au S.C.M. (Student Christian Movement, l'équivalent protestant de la J.E.C.). Pendant ces six mois, je me suis ouvert à toutes sortes de courants, qui étaient très vivement présents dans la conscience des jeunes chrétiens de cette génération. J'ai vécu au People's Palace, une sorte de commune avant la lettre, animée par le mouvement Quaker. Avec mes compagnons et compagnes au cœur de cette aventure, j'ai pratiqué certaines formes de mise en commun, de partage, et adopté certains rites de prière et de chant qui n'étaient pas ceux de notre tradition catholique. Je ne me rappelle plus bien si je fréquentais encore la liturgie catholique à ce moment. Mais mon souci de réfléchir sur la vie contemporaine à la lumière du patrimoine chrétien demeurait très vif. J'ai participé aux «work camps» organisés par le S.C.M. canadien et c'est là aussi que j'ai pris contact avec toutes sortes de mouvements effervescents, le mouvement trotskiste, sa tentative pour noyauter les syndicats mais aussi certaines expériences de psychiatrie avant-gardiste comme celles que l'on pratiquait à Webern en Saskatchewan, où dès les années 50, les hallucinogènes intervenaient dans le traitement psychiatrique.

Mais de cette époque, l'expérience la plus marquante aura sans doute été le séjour en usine chez Carboloy. C'est là qu'Yvan se familiarise avec la méthode de l'observation participante, en digne émule du William F. White de «Street's corner society». C'est là aussi que se nouent des amitiés avec certains jeunes intellectuels torontois: John Lee, Bob Wallenstein et maints autres, amitiés qui demeurent vives, même aujourd'hui.

Fin 1956, Yvan quitte Toronto et s'amène à l'École de service social de l'Université de Montréal. Vers la fin de leur séjour à l'Équipe nationale, les dirigeants de l'époque, Albert Breton, Raymond Breton, Jean-Paul Baillargeon, Maurice Pinard, Jean Fortier, Marc Lalonde en arrivent à la conclusion que l'on ne pourra pas faire sortir la pensée sociale au Québec de ses tendances à la "parlotte» et à la spéculation vide, sans adopter une attitude empirique et sans apprivoiser les disciplines et les méthodes qui permettraient de constituer des banques de données à partir desquelles une étude rigoureuse des réalités sociales serait rendue possible.

Albert Breton ira étudier l'économie à Colombia avec Stigler; Raymond Breton, lui, s'oriente vers la sociologie à l'Université de Chicago; Jean Fortier s'intéresse aux mathématiques appliquées et ira se former à Standford. Je me suis dit que si tout le monde partait on aurait au retour de sérieux problèmes d'enracinement, de réinsertion, et $\mathrm{j}$ 'ai décidé de rester. J'ai choisi aussi entre les deux pôles: recherche pure et dure d'une part, et souci d'action de l'autre, le pôle plus enraciné et plus soucieux de faire bouger les choses rapidement. Mon ami de toujours et premier modèle d'intellectuel engagé, Fernand Cadieux, m'avait fait remarquer qu'on pouvait devenir savant de deux façons: en s'expatriant quelques années pour aller étudier dans un grand centre, ou en se formant dans l'action, quitte à fréquenter, morceau par morceau, des lieux d'apprentissage comme des sessions de formation. Ce que j'aurai fait d'ailleurs toute ma vie par la suite. 
Yvan Corbeil arrive à l'École de service social à l'époque de la doctrine sociale de l'Église et de ses nombreux «a priori» désincarnés. Après son passage à la J.E.C. et au contact d'un homme qui a pris ses distances par rapport à de tels préjugés, Fernand Cadieux, Yvan ne se présente pas comme le candidat idéal pour cette école en symbiose parfaite avec les usages sociaux du temps, où seules les célibataires chez les femmes peuvent pratiquer le Service social et où une argumentation scolastique serrée tient lieu de référence empirique méthodique. Ce n'est qu'en se faisant discret, après qu'on l'eût averti que ses dangereuses mœurs (le travail en équipe, les voyages à Toronto ou à Washington) feraient de lui «une mauvaise tête qui ne ferait rien de bien», qu'Yvan, prenant son mal en patience, termine sa maîtrise. Sa thèse porte sur les premiers résultats d'une enquête menée conjointement avec deux collègues sur l'insertion des réfugiés hongrois à leur arrivée à Montréal.

J'avais déniché $300 \$$ au Conseil des œuvres de Montréal, que dirigeait à l'époque l'abbé Robert Riendeau.

Tout au long de ses études, Yvan a fréquenté assidûment le Groupe de recherche sociale et est devenu dans les faits, le spécialiste de l'échantillonnage et des procédures d'enquête. Au sortir de Service social, après quelques mésaventures au ministère de la Jeunesse, au service d'accueil aux voyageurs, il mesure jusqu'à quel point «un homme trop instruit et intellectuellement trop bien organisé, convient mal aux institutions de l'époque et à leurs plis idéologiques».

Nous sommes en 1959. Le dégel s'annonce au Québec mais dans beaucoup de secteurs, la rigidité traditionnelle est encore bien en place. C'est donc avec plaisir et soulagement, qu'Yvan accepte l'invitation qui lui est faite par Antonin Boisvert et Soucy Gagné qu'il a connus, le premier comme professeur à l'université, et le second à la J.E.C. universitaire, de se joindre au Service de recherche de Radio-Canada:

C'est vraiment là, à Radio-Canada, que j'ai appris mon métier. Nous avions les coudées franches et grâce au leadership tout à fait exceptionnel d'Antonin Boisvert, son souci de rigueur et de professionnalisme, nous avons pu nous mesurer aux standards les plus rigoureux de la recherche sociale. C'est là aussi que j'ai pris contact avec A.A.P.O.R. (American Association for Public Opinion Research) et avec W.A.P.O.R. (Word Association for Public Opinion Research) qui auront été tout au long de ma vie, d'importantes sources d'alimentation et qui $\mathrm{m}$ 'auront permis d'élaborer un réseau de relations nationales et internationales dont j'aurai tiré toutes sortes de bénéfices. C'est au moment où je suis à Radio-Canada que je prends contact avec des intellectuels canadiens-anglais, américains et européens représentant de manière émérite les Sciences sociales appliquées.

La société québécoise a longtemps vécu repliée sur elle-même. Ceci laisse des traces à l'intérieur de la Société Radio-Canada. Les échanges entre le Service de recherche et l'extérieur sont assez rares. Yvan se donne alors comme rôle de favoriser l'ouverture. Il veut que le Service de recherche fonctionne en référence à la communauté scientifique internationale. Il participe à des rencontres scientifiques, prend contact avec des homologues américains, européens, canadiens-anglais. Certains comme Jean Stoetzel deviendront de grands amis; d'autres viendront en visite à Radio-Canada. Dans les débats sur la forme à donner à ce service de recherche, Yvan favorise l'adoption d'un modèle «recherches sociales» dont le mandat implique beaucoup plus qu'un service de recherche sur l'auditoire. Il préfère de loin le mandat plus large qui ménage plus de liberté aux chercheurs: ils font plus que de remplir des commandes d'études et de sondages des auditoires, telles que formulées par les responsables. Il faut que les chercheurs puissent influencer les diffuseurs à partir de problématiques de longue haleine sur les rapports entre la société et les mass media. Il pense aussi qu'un service de recherche ferait mieux de loger haut dans la hiérarchie,

constituer une vice-présidence, par exemple, de manière à ce que les chercheurs puissent influencer les orientations à long terme, et se laisser eux-mêmes atteindre par les préoccupations et les interrogations des décideurs aux plus hauts niveaux.

Mais après quelques années, je me rends compte que le type de recherches que je voudrais favoriser, n'est pas facile à réconcilier avec les règles opérationnelles d'une bureaucratie de la taille de Radio-Canada. Je pense, par exemple, à la «recherche éclair» 
qu'il aurait fallu faire pendant la grève du journal La Presse. Je pense encore à cette communication à un des premiers congrès de W.A.P.O.R. (World Association for Public Opinion Research) auquel je voulais participer mais qu'il aurait fallu soumettre d'abord aux instances suprêmes de Radio-Canada avant d'aller au congrès.

Soucy Gagné et Yvan, quelques autres collègues aussi, réfléchissent et arrivent rapidement à la conclusion qu'ils ne pourraient réaliser un certain type de recherches sociales à l'intérieur de la Société Radio-Canada, si libérale soit-elle, comparativement aux autres institutions canadiennes-françaises et québécoises de l'époque. Il n'y aura vraiment d'autres recours que de créer une institution privée, plus flexible, et laissant des marges de manœuvre optimales aux chercheurs.

\section{CROP: DES SONDAGES D'OPINIONS AUX SUIVIS SOCIO-CULTURELS.}

En 1965, Yvan Corbeil fonde C.R.O.P. Il aurait préféré faire de C.R.O.P. une entreprise d'équipe. De la J.E.C. au Groupe de recherche sociale à Radio-Canada, Yvan aura toujours été un homme d'équipe. Mais aucun de ses collègues de Radio-Canada n'est prêt à sauter dans le bateau avec lui.

Je pense que mes collègues ne voyaient pas en moi un leader et un chef d'entreprise.

Yvan est déçu d'avoir à faire seul ce mouvement, parce qu'il craint de répéter le modèle de sa famille où tous les pères de quatre générations ont été des chefs d'entreprise prospères dans le secteur de la chaussure et ont tous fait de leur «affaire» un «one man show». Consulté sur le projet, Fernand Cadieux veut l'en dissuader: «Tu devrais faire au moins une étude de marché, Yvan!». Cette fois, l'ami et le mentor aura été de mauvais conseil et c'est donc seul, qu'Yvan se lance dans l'aventure. Après de nombreuses conversations avec Janine, sa femme, il décide d'un emprunt à la banque et incorpore C.R.O.P. pour Centre de recherche sur l'opinion publique.

$\mathrm{Au}$ début, première phase, les travaux consistent essentiellement en études ad hoc par opposition à des recherches récurrentes. La Commission Larendeau-Dunton est une pourvoyeuse importante de contrats. On trouve aussi parmi les tous premiers clients, l'Alliance des professeurs, la Commission des écoles catholiques de Montréal, la Fédération des œuvres. Très tôt, Yvan concède des actions minoritaires dans C.R.O.P. aux mathématiciens à la Société de mathématique appliquée (S.M.A.) dont il acquiert lui-même des actions. Un peu plus tard, Soucy Gagné quitte Radio-Canada et se joint à la Société de mathématiques appliquées. Dans les faits, il est le principal collaborateur d'Yvan pour tout ce qui concerne la recherche sociale. En peu de temps, c'est le secteur des recherches sociales, et pas uniquement les sondages d'opinions, qui devient la locomotive de la Société de mathématiques appliquées. Les mathématiciens ont de la difficulté à se trouver des contrats à cette époque, beaucoup plus que les chercheurs du secteur social.

En 1968, la Société de mathématique appliquée vend des actions sur le marché public et à ce moment-là les mathématiciens sont majoritaires. Le mariage C.R.O.P.-S.M.A. n'aura duré que quelques années. Yvan quittera le giron de S.M.A. pour se consacrer uniquement à C.R.O.P. qui était devenu entre-temps, une entreprise bien établie où l'on trouve déjà un personnel d'une dizaine de chercheurs engagés à temps plein, très majoritairement psychologues ou sociologues. Soucy Gagné, de son côté, avait déjà quitté S.M.A. pour fonder S.O.R.E.C.O.M.

De tout temps, Yvan Corbeil se définit comme un chercheur, et non comme un sondeur d'opinions, au sens restreint du terme. Il crée des projets de recherche et tente d'intéresser ses clientèles à des questions de recherche de plus longue haleine qui supposeraient des analyses récurrentes plutôt que des instantanés synchroniques. Mais les clientèles, autant privées que publiques, se font tirer l'oreille! Ce n'est qu'en 1976 qu'Yvan créera le Bulletin C.R.O.P. Â partir de là, on pourra procéder à des analyses de tendances. Les sondages omnibus posent un train de questions, plusieurs fois par année, année après année. Ces questions permettent d'établir dans quelles directions changent les mentalités à propos de valeurs comme la santé, le travail, la sexualité, les habitudes de consommation, etc. Le bulletin C.R.O.P. rejoint 
plusieurs clientèles intéressées à un cœur d'informations commun, auquel se greffent des informations spécifiques à chacun et auxquelles le destinataire possède un accès confidentiel.

Ce sont d'abord les ministères anglophones du Canada qui ont appuyé le Bulletin C.R.O.P. et qui m'ont permis de quitter les études «ad hoc» ou du moins de leur donner moins de place comparativement à des recherches de plus longue haleine. Les abonnés au Bulletin C.R.O.P. reçoivent régulièrement des données sur l'évolution des tendances, année après année, au Québec et au Canada. Plus tard, grâce à mes contacts internationaux via W.A.P.O.R. nous créerons des postes d'observation des suivis socio-culturels et mettrons au point un système (T.R.I.-S.C.) qui depuis 1983 permet des études comparatives à travers dix-huit pays différents sur l'évolution des valeurs et des mentalités au sein des sociétés contemporaines. Maintenant que nous publions dans chacun des dix-huit pays membres, année après année, un rapport annuel fait par chacun des centres d'observation des suivis socio-culturels, nous sommes en mesure de fabriquer des scénarios de développement à partir des grandes valeurs autour desquelles les populations se différencient et évoluent, qu'il s'agisse de la mode, de l'alimentation, des mœurs sexuelles et familiales, des comportements politiques, etc...

Ces recherches menées dans dix-huit pays, en Amérique du Nord et du Sud, en Europe, en Asie, au Moyen-Orient et en Afrique, nous permettent de constituer annuellement une carte socio-culturelle des courants par lesquels passent les diverses sociétés. Cela rend possible de situer chacune des sociétés par rapport à trois grands axes. Premièrement, la préséance du conformisme par opposition à l'informalité et à la permissivité; deuxièmement, la place accordée à l'affirmation de soi pouvant aller jusqu'au repli sur soi; troisièmement, la valeur accordée au plaisir et à la liberté par opposition à l'ascèse et à l'effort. On peut par exemple, constater mondialement, une forte tendance vers «l'informalité» dans l'ensemble des sociétés de méme qu'une montée des valeurs reliées à l'affirmation de soi. Ce qui n'empêche pas qu'à l'intérieur de chacune de ces sociétés, tantôt sur des bases régionales, tantôt à partir d'autres clivages, on puisse identifier un très grand nombre de sous-groupes, un peu comme si toutes les scieiétés se morcelaient en unités plus petites possiblement très différenciées. Bien sûr, la capacité de comparer la situation, année après année, entre dix-huit pays différents, et plus subtilement à l'intérieur d'un rêpertoire qui comporte un grand nombre de variations régionales, permet de construire des scénarios pour chacune de ces sociétés (généralement plusieurs) et de voir venir dans quelles directions le changement social s'oriente. Bien sûr les décideurs ont intérêt à braquer leur lunette sur ces grands courants. Ces décideurs, on les trouve à RadioCanada, à l'Hydro-Québec, dans les ministères, mais aussi dans les entreprises privées de l'envergure de Shell, de Bell, d'Alcan et de nombreuses autres.

Plus profondément, la pratique de la recherche de plus longue haleine mène à une nouvelle conception de la relation de consultation. D'une part, les clients sont invités à envisager le développement de leur entreprise dans une perspective beaucoup plus ample, mondiale et historique. Un autre aspect important, c'est le changement apporté par Yvan Corbeil et ses collègues de divers pays, particulièrement la France, l'Italie, l'Angleterre, à la manière de poser, l'un par rapport à l'autre, le terme subjectif de ce que le décideur souhaite, imagine, préfère, et le terme objectif, qui lui, ressemble plus à une photographie beaucoup plus précise de la situation. C'est là une véritable révolution dans l'utilisation du savoir à des fins de changement social. On en arrive ainsi à réconcilier le constat objectif rigoureux et le monde de l'intuition, de l'affect, de l'imaginaire, à l'extrême même, du vécu corporel. Avec certains collègues de France, d'Italie, d'Angleterre et de Suisse, Yvan a créé une firme qui s'appelle R.I.S.C. (Research on International Socio-cultural Trends) qui gère, depuis son secrétariat en Suisse, l'implantation internationale du système T.R.I.-S.C.

JANINE ET LA GESTALT: LE RENOUVEAU DU PARADIGME DE L'ENQUÊTE SOCIALE.

Yvan Corbeil et Janine Gagnon se sont rencontrés lorsqu'ils étudiaient tous les deux à l'Université de Montréal. 
J'étais dans l'autobus boulevard St-Joseph quand j'ai rencontré Janine qui accompagnait Aimée Leduc, une ancienne de la Centrale. C'est donc Aimée qui m'a présenté à Janine.

Janine et Yvan se sont rencontrés un certain nombre de fois, mais elle est partie pour Paris, étudier en psychologie aux termes de sa maîtrise à l'Université de Montréal. Ce n'est qu'à son retour en 1960 qu'ils se retrouveront:

Nous nous sommes fréquentés six mois, puis encore six mois et nous étions mariés. Je me rappelle que nous nous sommes fiancés dans le train qui nous menait chez les parents de Janine à Âmqui.

Yvan, même à l'époque la plus effervescente de C.R.O.P., s'est toujours arrangé pour accorder une priorité importante à sa vie amoureuse et familiale.

J'ai très rarement travaillé les week-ends et j'ai investi beaucoup de temps et d'énergie dans ma vie de couple et familiale. Janine connaissait plusieurs écoles de psychologie; elle était familière de la psychanalyse kleinienne par exemple, à une époque où on connaissait peu ce mouvement de pensée. Mais c'est sa rencontre de la GESTALT, à Cleveland en 1972, qui aura été pour elle un tournant majeur, non seulement de sa vie professionnelle, mais aussi de sa vie familiale.

La GESTALT est une école psychothérapeutique fondée par Fritz Perls, appartenant au grand courant de la psychologie existentielle et humaniste. Le thérapeute aide le client à devenir conscient de son vécu émotionnel, corporel, imaginaire, intellectuel. Il est généralement plus actif qu'un psychanalyste ou un thérapeute centré sur le client. Il lui arrive souvent de proposer des exercices, des jeux dramatiques ou autres formes de cadres d'apprentissage pour en arriver à favoriser chez son client des prises de conscience aptes à le conduire à la fermeture de certaines «gestalten» (d'où le nom de l'école) - c.-à.-d. de certains «touts» expérientiels dont l'intégration est bénéfique au développement de la personne.

Quand Jeannine reviendra de cette session, Yvan vivra comme un choc la nouvelle manière dont elle entendra se définir comme femme dans son rapport avec lui, son conjoint, mais aussi avec la famille et le travail. C'est pour mieux comprendre sa femme et garder contact avec elle qu'Yvan s'intéressera à la GESTALT. Il ira lui-même, en 1974, s'initier à la GESTALT à Cleveland, et tout au long des années 74 et 75 et par la suite, il se soumettra à divers apprentissages (groupe de thérapie pour hommes, formation sur mesure à la «gestalt» et travail avec de multiples approches corporelles: Feldenkreiss, Alexander, Lomi). La méthode Feldenkreiss entend favoriser la prise de conscience par le mouvement corporel: en différenciant les stratégies du mouvement corporel, on atteint les centres supérieurs du cerveau, eux aussi soumis à une différenciation plus poussée. La méthode Alexander se préoccupe surtout de la posture physique et de sa signification pour le fonctionnement physique et psychique. Pour sa part, la méthode Lomi veut libérer l'énergie (en cela elle est dérivée de la bio-énergie et s'apparente au «rolfing») en appliquant des pressions par massage sur l'enveloppe des muscles. Contrairement au «rolfing», il s'agit de pressions douces qui visent un assouplissement progressif de la musculature.

Yvan travaillera aussi avec Milton Erickson le grand maître de l'hypnose. Les thérapeutes gestaltistes chevronnés comme Downa Markova et Llena Rubenfeld lui apporteront également beaucoup, personnellement et professionnellement.

Devenu un animateur gestaltiste en bonne et due forme, Yvan n'en demeure pas moins un chercheur social et le principal leader de C.R.O.P. En même temps qu'il acquiert sa nouvelle formation psychologique, il travaille à la mise au point du système T.R.I.-S.C., et au plan international constitue le réseau qui, plus tard, lui permettra, avec certains collègues, Alain DeVulpian en particulier, de créer R.I.S.C. et d'opérationnaliser les suivis des courants socio-culturels.

La synthèse la plus nette entre le chercheur social et l'intervenant gestaltiste prendra la forme de séminaires d'un nouveau genre qu'il offre aux principaux clients de C.R.O.P., hauts fonctionnaires, éducateurs, entrepreneurs privés, etc. Dans ces séminaires, Yvan utilise diverses techniques (hypnose, fantaisie dirigée, etc.) pour faire prendre conscience aux décideurs que leur univers subjectif est en cause, qu'il ne s'agit pas uniquement et simplement de scruter à la loupe le tissu social avant d'entreprendre une action pertinente. Cette dialectique, l'étude 
la plus poussée possible, la plus empiriquement fondée des faits sociaux, en même temps que la réflexion sur soi et l'écoute de messages d'un autre genre (états corporels, rêves, fantaisies) permet un renouveau du paradigme de l'enquête sociale. Yvan a fait de nombreuses conférences sur ce sujet, écrit des textes remarquables et au moment d'arriver à sa maturité, il propose une pensée originale qui réconcilie le regard vers l'extérieur et l'écoute de soi dans la production d'un nouveau type d'hypothèses, dont la fécondité pour l'action et le développement personnel paraît tout à fait exceptionnelle.

En trente ans, Yvan aura traversé une époque extrêmement turbulente, aussi bien au plan des valeurs professionnelles qu'au plan des valeurs personnelles et familiales. D'une crise à l'autre, d'une recherche à l'autre, il sera demeuré fidèle à lui-même, en réconciliant les facettes nombreuses et vives d'une personnalité riche et complexe, véritable kaléidoscope organisé autour de quelques axes fondamentaux; patiente fidélité et histoire de toute une vie; la recherche et l'ouverture à plusieurs points de vue, l'amour et l'affection, le sens de l'aventure, les vastes horizons géographiques et culturels.

\section{LA MALADIE}

Seule la maladie, et encore, aura ralenti une telle énergie et un tel besoin d'accomplissement. La sclérose amyotrophique latérale est une maladie bien connue mais devant laquelle la médecine s'est montrée jusqu'ici impuissante. Son progrès est redoutable. De trois mois en trois mois, Yvan voit sa motricité diminuée et il doit, au quotidien, compter avec une baisse d'énergie importante.

Il est très difficile de s'adapter à la vie d'une personne handicapée qui évolue principalement en chaise roulante. J'ai dû réaménager ma maison et me faire construire un ascenseur personnel. Je dois fréquenter uniquement les restaurants qui comportent une pente douce à l'entrée, je ne peux plus conduire mon automobile, j'ai plus de difficultés qu'avant à calligraphier, etc.

C'est devant les grands problèmes de l'existence qu'une personne exprime sa fibre véritable. Son comportement dans de tels contextes, (la maladie, la souffrance, la mort d'un proche) fait valoir, exprime ce qu'elle est en profondeur. La manière dont Yvan réagit à sa maladie est une véritable «signature». Son comportement dans ce contexte reprend l'essentiel de ce qu'il a toujours été. D'abord la recherche: à l'heure actuelle, Yvan Corbeil est probablement la personne la mieux renseignée au monde sur la sclérose amyotrophique latérale. Il est en contact avec des dizaines de praticiens de toutes sortes de médecines. De la même manière qu'il ne s'est pas laissé coincer dans les préjugés d'époque qu'il a dû dépasser pour devenir le chercheur qu'il est, il s'est permis des incursions dans toutes sortes de médecines autres que la neurologie établie dont son cas relève officiellement. Recherche et ouverture, curiosité infatigable mais aussi relations avec les autres, action concertée et souci de créer des institutions viables. Yvan est maintenant coordonnateur de l'Association de la sclérose amyotrophique latérale; à l'Institut de réadaptation de Montréal où il doit suivre régulièrement des traitements, il est devenu membre du Conseil d'administration et travaille à la mise au point d'un programme de recherche clinique qui fait cruellement défaut dans ce secteur, de manière à ce que les victimes actuelles et éventuelles de ce mal terrible puissent compter sur des appuis médicaux plus riches que ce que la tradition véhicule jusqu'ici. Un peu partout au Canada anglais, aux États-Unis et ailleurs dans le monde, des collègues qu'Yvan a connus alors qu'il a été président de W.A.P.O.R. et avec lesquels il a travaillé à mettre sur pied le réseau R.I.S.C. s'offrent à créer un fonds de recherche et une association internationale pour faire avancer la connaissance sur ce mal mystérieux et impitoyable.

Comme pour tous les autres aspects de sa vie, Yvan porte sur sa maladie un regard polyvalent, ouvert, où les ressources de la recherche expérimentale sont à conjuguer avec celles du suivi clinique mais aussi avec des apports traditionnels, médecine orientale, médecine douce et attitudes spirituelles face à la souffrance, à la maladie et éventuellement à la mort.

Là comme ailleurs la traversée d'Yvan Corbeil est exemplaire. Tous ceux qui le côtoient retrouvent en lui un sage, un témoin et par-dessus tout, un homme capable d'une immense 
affection pour ses semblables, peu importe ce qui les différencie, qu'ils déambulent à pieds ou qu'ils doivent faire leur chemin en chaise roulante. Au bout de son âge, Yvan Corbeil retrouve de vieilles complicités avec le monde de la spiritualité: la chrétienne, souvent d'ailleurs protestante: Karl Barth, Paul Tillich; mais aussi l'orientale. Il médite plusieurs heures par jour, voit beaucoup d'amis et continue d'espérer.

Au sommet d'une carrière complexe et qui a foisonné en toutes sortes d'événements significatifs, à quel type de conclusions arrive-t-on?

Il faut réinventer le contrat qui relie le décideur et le chercheur. Les chefs d'entreprise ont tout à perdre à s'entourer de chercheurs qui n'ont pas d'idées personnelles et qui se contentent d'exécuter des commandes. Mais il faut aussi que le chercheur apprenne à valoriser l'intelligence et l'imagination de ses clients. Les responsables et les chercheurs ont tout à gagner à une fécondation réciproque. Structurellement, il faudra d'ailleurs créer de nouvelles fonctions pour que la recherche cesse d'être une officine en périphérie et devienne une valeur centrale, logée très haut dans les hiérarchies, peut-être autour d'un poste comme une vice-présidence à la recherche par exemple. Les entreprises qui s'adaptent mieux à l'époque sont justement celles qui sont soucieuses de recherche à long terme et qui voient plus loin que la mise en marché de leur prochain produit. La lecture que j'ai faite de la table ronde (dans le présent numéro) me convainc de certaines choses. Plusieurs des sociologues de la génération actuelle ont une perspective de temps trop courte. Souvent, ils ne sont pas au fait de ce qui s'est passé dans leurs propres institutions, il y a vingt ans par exemple. Ils entretiennent aussi sur l'entreprise privée un certain nombre de préjugés communs, à savoir qu'elle est constamment soucieuse de rentabilité à court terme et incapable d'envisager des problématiques de longue haleine. L'histoire de ma vie m'aura convaincu de l'inverse. Les entreprises les plus saines sont celles qui sont capables de l'envergure qu'il faut pour saisir un contexte global, c'est-à-dire planétaire et prospectif; et on trouve des administrateurs de ce calibre autant dans l'entreprise privée que dans l'entreprise publique.

Yvan est convaincu que les universités gagneraient à s'associer de plus près au monde des affaires et à la haute fonction publique, à l'intérieur de structures multilatérales, au lieu de perpétuer la tradition de retrait qui souvent mène à l'anachronisme.

Cela aura sans doute été une erreur de créer à l'Université de Montréal un centre de sondage qui est maintenant fermé et qui a mené une concurrence douteuse aux boîtes privées, dont C.R.O.P. Par contre, il serait extrêmement important que les départements de sciences sociales se préoccupent plus de relectures et d'analyses secondaires. Et c'est assez naturellement à l'université, que devrait loger un centre de recherches sur l'évolution des tendances socio-culturelles essentiellement nourri d'analyses de deuxième ordre à partir du flot considérable de sondages et d'enquêtes pratiqués à travers le monde.

Yvan Corbeil a suffisamment vécu pour que l'on puisse se demander quels grands traits de sa personne révèle sa biographie. Contrairement à beaucoup de ses contemporains, Yvan n'aura pas eu à choisir entre une carrière brillante et une vie de famille riche. Il a fait l'une et l'autre et probablement l'une par l'autre. Le chalet dans les Laurentides, les voyages, les rites et les fêtes de la vie privée auront été un précieux ressourcement et le compagnonnage de Janine, une référence intellectuelle majeure tout au long de sa vie.

Plusieurs grandes valeurs auront marqué cette existence exceptionnelle. D'abord et avant tout la liberté! C'est elle qui l'aura placé en porte-à-faux à peu près partout; à la J.E.C. au Service d'accueil aux voyageurs, à l'École de service social et même d'une certaine manière à Radio-Canada. C'est elle, aussi, qui l'aura convaincu de la nécessité de se donner à luimême l'instrument requis par l'action dont il rêvait: le Centre de recherches sur l'opinion publique. Mais c'est encore elle qui lui aura fait dépasser la vocation première d'une «boîte de sondages» pour créer un instrument de recherche extrêmement complexe et dont la portée, tant pour la recherche que pour l'action, s'avère très importante, nous parlons ici du système T.R.I.-S.C., des suivis socio-culturels.

Yvan aura dû faire beaucoup de choses par lui-même! Il s'est donné en service social un programme de formation ad hoc. Il a appris en la pratiquant, et en fréquentant des stages à l'Université du Michigan, l'essentiel de la méthodologie de l'enquête. Son programme de 
formation gestalt aura été aussi un programme de formation sur mesure. À travers vents et marées, Yvan aura porté jusqu'à ses ultimes conséquences sa singularité comme personne. C'est à travers elle qu'il rejoint, au déclin de la vie, dans la force de l'âge à 53 ans, certains grands thèmes universels. Depuis quelques années, Yvan s'est beaucoup soucié de développer la partie féminine de sa personnalité, sans perdre pour autant les traits de l'homme qu'il a toujours été. Mêlant le besoin de s'enraciner dans son pays à une envergure internationale remarquable; restant fidèle à certaines valeurs mais toujours capable de les reconnaître en profondeur sous les atours que l'époque leur donne. Contrairement à la très forte majorité des intellectuels de sa génération, Yvan n'a aucun préjugé par rapport à l'entreprise privée.

Il ne s'agit pas d'être pour ou contre l'entreprise privée. Il s'agit dans chaque cas de décider des moyens adéquats pour servir une action valorisée pour elle-même et pour les buts à long terme qu'elle permet d'atteindre.

Là où en est Yvan dans sa traversée, on commence à soupçonner de quoi est faite l'autre rive. Depuis la profondeur de sa solitude et de sa lucidité, cet homme qui aura négocié si admirablement une époque si difficile, qui se sera libéré de contraintes institutionnelles extrêmement sévères et qui aura perdu des illusions pour trouver des valeurs, cet homme aura encore beaucoup de choses à nous dire! 